\title{
O estado da arte da biodiversidade de rotíferos planctônicos de ecossistemas límnicos de Pernambuco
}

\author{
Mauro de Melo Júnior ${ }^{1,4}$, Viviane Lúcia dos Santos Almeida ${ }^{2}$, \\ Sigrid Neumann-Leitão ${ }^{3}$, Maryse Nogueira Paranaguá ${ }^{2} \&$ Ariadne do Nascimento Moura $^{2}$ \\ Biota Neotropica v7 (n3) - http://www.biotaneotropica.org.br/v7n3/pt/abstract?article + bn01707032007 \\ Recebido em 21/05/07 \\ Versão reformulada recebida 06/08/07 \\ Publicado em 17/09/07 \\ ${ }^{1}$ Departamento de Oceanografia Biológica, Instituto Oceanográfico, Universidade de São Paulo - USP, \\ CEP 05508-900, São Paulo, SP, Brasil, www.io.usp.br \\ ${ }^{2}$ Departamento de Biologia, Universidade Federal Rural de Pernambuco - UFRPE, \\ CEP 52171-900, Recife, PE, Brasil, www.ufrpe.br \\ ${ }^{3}$ Departamento de Oceanografia, Universidade Federal de Pernambuco - UFPE, \\ CEP 50670-901, Recife, PE, Brasil, www.ufpe.br/docean \\ ${ }^{4}$ Autor para correspondência: Mauro de Melo Júnior, e-mail: mmelojunior@gmail.com
}

\begin{abstract}
Melo Júnior, M., Almeida, V.L.S., Neumann-Leitão, S., Paranaguá, M.N. \& Moura, A.N. State of the art in planktonic rotifers biodiversity from freshwater environments in the state of Pernambuco (Brazil). Biota Neotrop. Sep/Dez 2007 vol. 7, no. 3 http://www.biotaneotropica.org.br/v7n3/pt/abstract?article+bn01707032007. ISSN 1676-0603.

The state of the art in planktonic rotifers biodiversity from some freshwater ecosystems was elaborated for the state of Pernambuco (Brazil). A bibliographic survey was done and most studies focused seasonal aspects and trophic state of the ecosystems. At least 19 continental aquatic environments related to rotifer researches have been studied. The Sertão area was the most studied. The present survey registered 64 species of planktonic rotifers, distributed in 18 families and 29 genera. The most frequent species were Keratella tropica and Brachionus falcatus, showing that most studies were done in the limnetic zone. The results showed the need for more research on the biodiversity of this group in ecosystems located in Zona da Mata and Agreste areas, as well as in lotic systems and in the littoral zones, in view of the lack of information.

Keywords: Rotifera, check-list, limnic ecosystems, Pernambuco, northeast Brazil.

\section{Resumo}

Melo Júnior, M., Almeida, V.L.S., Neumann-Leitão, S., Paranaguá, M.N. \& Moura, A.N. O estado da arte da biodiversidade de rotíferos planctônicos de ecossistemas límnicos de Pernambuco. Biota Neotrop. Biota Neotrop. Sep/Dez 2007 vol. 7, no. 3 http://www.biotaneotropica.org.br/v7n3/pt/abstract?article+bn01707032007. ISSN 1676-0603.

Este trabalho apresenta uma avaliação do estado da arte da biodiversidade de rotíferos planctônicos de alguns ecossistemas aquáticos continentais para Pernambuco (Brasil). Um levantamento bibliográfico foi realizado e a maior parte das pesquisas enfocou aspectos sazonais e estado trófico dos ecossistemas. Pelo menos 19 locais foram estudados no tocante aos rotíferos. O Sertão é a região mais estudada. Nesta pesquisa foram registradas 64 espécies de rotíferos planctônicos, distribuídas em 18 famílias e 29 gêneros. As espécies mais freqüentes nos locais estudados foram Keratella tropica e Brachionus falcatus, mostrando que a maior parte dos estudos foi realizada na zona limnética. Os resultados sugerem ainda a necessidade de mais pesquisas sobre a biodiversidade deste grupo em locais localizados na Zona da Mata e no Agreste, bem como em ecossistemas lóticos e nas zonas litorâneas dos locais, tendo em vista a carência de informações.
\end{abstract}

Palavras-chave: Rotifera, lista de espécies, ecossistemas límnicos, Pernambuco, nordeste do Brasil. 


\section{Introdução}

Os rotíferos ocorrem preferencialmente em ecossistemas aquáticos continentais, apresentando grande diversidade de formas (Schäefer 1985). A reprodução destes organismos é partenogenética, com ciclo de vida curto, podendo formar ovos de resistência (Barnes et al. 1995, Sipaúba-Tavares \& Rocha 2001) ou apresentar variações sazonais nas taxas reprodutivas associadas à variabilidade da disponibilidade alimentar (Devetter \& Sed' A' 2003). Este grupo é elo fundamental na teia trófica aquática, alimentando-se de algas microscópicas e bactérias, cobrindo o nicho ecológico dos pequenos filtradores (Hutchinson 1967, Nordy \& Watanabe 1978, Margalef 1983, Bonecker \& Aoyagui 2005). Os rotíferos são largamente utilizados na aqüicultura como alimento para formas jovens de peixes e crustáceos (Neumann-Leitão \& Souza 1987) e destacam-se também como bioindicadores das condições tróficas das águas (Berzins \& Pejler 1989, Neumann-Leitão et al. 1989, Güntzel \& Rocha 1998, Duggan et al. 2001, Nogueira 2001).

Apesar de o estado de Pernambuco fazer parte do conhecido "Polígono das Secas" no Nordeste do Brasil, o mesmo possui alta riqueza de ambientes límnicos. Porém, desde os primeiros relatos sobre a escassez de pesquisas realizadas até o final da década de 1980 (Neumann-Leitão et al. 1989), os estudos enfatizando a comunidade zooplanctônica em Pernambuco mostram poucos avanços nos dias atuais. Além disso, uma relevante parcela destes estudos faz parte da chamada literatura cinza (relatórios técnicos, monografias, dissertações e teses), disponível apenas em algumas bibliotecas e acervos públicos e privados, muitas vezes de difícil acesso aos pesquisadores.

O presente trabalho consiste no primeiro levantamento de espécies de rotíferos planctônicos de ambientes aquáticos continentais de Pernambuco. Diversos tratados sobre a biodiversidade têm apontado que a elaboração de base de dados biológicos deve ser constantemente incentivada como parte dos esforços para melhor direcionamento dos programas de conservação da biota e dos ecossistemas (Groombridge 1992, Heyhood 1995). Desta forma, os objetivos principais deste trabalho são (1) inventariar a biodiversidade de rotíferos planctônicos dos ecossistemas continentais de Pernambuco, bem como o de (2) sintetizar o estado atual de conhecimento acerca da biodiversidade destes organismos.

\section{Material e Métodos}

O inventário de rotíferos planctônicos de ecossistemas continentais do estado de Pernambuco foi obtido a partir da literatura disponível em periódicos, monografias, dissertações, teses, relatórios técnicos e trabalhos ainda não publicados. Vale ressaltar que não foram considerados resumos divulgados em eventos científicos. Após o levantamento, os trabalhos foram analisados e classificados por temas abordados, de acordo com os assuntos tratados nos textos. Alguns trabalhos foram catalogados em mais de um tema. Cada uma das citações recebeu um número de acordo com a ordem cronológica dos anos de publicação, o qual é utilizado nas tabelas como referência (ver Tabela 1).

Quanto aos locais aquáticos apontados na literatura, estes foram enquadrados em quatro regiões do estado de Pernambuco: Região Metropolitana do Recife (RMR), Zona da Mata, Agreste e Sertão. Ao todo, a fauna de rotíferos planctônicos foi estudada em pelo menos 19 locais de água doce (um deles em dois momentos), localizados sobretudo no Sertão. Para cada um destes locais são apresentadas, quando disponíveis, informações referentes às coordenadas geográficas, cidade, estado trófico, malha da rede e período de coleta (Tabela 1).

Após a revisão, as espécies foram listadas de acordo com a classificação proposta por Segers (2002) e separadas por família e gênero. Em seguida, foi feito um cálculo de frequiência de ocorrência (Fr) com base na distribuição de cada espécie nos locais estudados: $\operatorname{Fr}(\%)=\left(\mathrm{Oc}^{*} 100\right) / 20$, onde Oc: número de ocorrências das espécies nos locais estudados. Após os cálculos, foram consideradas como freqüentes as espécies com Fr maior ou igual a 50\%, acessórias as espécies com Fr maior ou igual a $25 \%$ e menor que $50 \%$ e acidentais as espécies com Fr menor que 25\%. Algumas espécies foram citadas mais de uma vez para um mesmo ambiente, porém elas foram consideradas apenas uma vez para cada ambiente nos cálculos de freqüência. Convém mencionar que não foram consideradas nestes cálculos as citações apenas de morfotipos (por exemplo: Rotaria sp. ou Rotifera sp. 1).

\section{Resultados e Discussão}

As pesquisas sobre rotíferos planctônicos de ambientes aquáticos continentais de Pernambuco tiveram início no Sertão do Estado com Ahlstrom (1938). Neste trabalho, foram citadas apenas duas espécies (Brachionus plicatilis - muito provavelmente Brachionus rubens - e Lacinularia striolata). Entretanto, os primeiros grandes levantamentos de espécies foram realizados apenas na década de 1980, com os trabalhos de Neumann-Leitão (1981), Paranaguá \& Neumann-Leitão (1982), Neumann-Leitão \& Nogueira (1986), Neumann-Leitão \& Souza (1987), Neumann-Leitão \& Nogueira-Paranhos (1987/89) e Neumann-Leitão et al. (1989), sobretudo estes três últimos. Com exceção do trabalho de Neumann-Leitão \& Nogueira-Paranhos (1987/89), os demais foram realizados na RMR, incluindo viveiros de cultivo de camarão de água doce e o Açude de Apipucos, construído antes de 1900 com o objetivo de amenizar as enchentes no rio Capibaribe.

Os estudos sobre os rotíferos límnicos de Pernambuco foram retomados apenas em 2000, com o trabalho de Crispim \& Watanabe (2000). Neste trabalho, foram estudados quatro ecossistemas, incluindo dois ambientes lóticos (rio Brígida e uma parte do rio São Francisco). Em seguida, uma série de trabalhos foi desenvolvida nas quatro regiões do estado de Pernambuco (Bouvy et al. 2001, Almeida 2002, Melo Júnior 2002, Moura 2004, Almeida et al. 2006, Melo Júnior, dados não publicados), todos em reservatórios.

Quanto aos temas abordados nos trabalhos sobre rotíferos planctônicos, destacam-se a sazonalidade e o estado trófico dos ambientes, com $81,25 \%$ e $75,00 \%$ dos trabalhos, respectivamente (Tabela 2). Apesar de a sazonalidade ter sido bastante abordada nos trabalhos, poucos apresentaram a variação dos rotíferos em ciclo anual completo, sendo registrada geralmente a distribuição dos rotíferos apenas em alguns meses dos períodos seco (setembro a fevereiro) e chuvoso (março a agosto). Na literatura consultada foram também abordados a variação espacial, índices de diversidade específica, eqüitabilidade, variação nictimeral, zona litorânea e distribuição vertical. Dentre esses, a variação nictimeral e coletas em zonas litorâneas foram citados em apenas $25,00 \%$ da bibliografia consultada. Caso esses dois assuntos fossem tratados nas demais pesquisas já realizadas em Pernambuco, muito provavelmente o número de espécies aumentaria consideravelmente, tal como foi relatado por estudos que contemplaram tais aspectos (Almeida et al. 2006, Almeida, dados não publicados).

Ao todo, a fauna de rotíferos planctônicos foi estudada em 19 locais de água doce (rios, reservatórios, açudes, viveiros e poços d'água). O Sertão apresentou maior número de locais estudados (9), seguido pela RMR (5), Agreste (3) e Zona da Mata (2). Os ecossistemas lênticos são os mais bem estudados, sobretudo os reservatórios ou açudes, com um total de 15 . Por outro lado, apesar de serem comuns no bioma Caatinga, os lagos temporários ainda não foram objeto de estudo no que diz respeito a esse grupo de animais. Quanto aos ecossistemas lóticos de Pernambuco, os estudos sobre rotíferos 
Tabela 1. Lista dos ambientes aquáticos continentais de Pernambuco já estudados quanto aos rotíferos planctônicos. Coord.: coordenadas geográficas. Ref.: referência bibliográfica N.I.: não indicado.

Table 1. Checklist of surveyed freshwater environments from the state of Pernambuco for planktonic rotifers. Coord.: geographic coordinates. Ref.: bibliographical references; N.I.: not indicated.

\begin{tabular}{|c|c|c|c|c|c|c|}
\hline Ambientes & Coord. & Cidade & $\begin{array}{l}\text { Estado } \\
\text { Trófico }\end{array}$ & Ref. & $\begin{array}{c}\text { Malha } \\
(\mu \mathrm{m})\end{array}$ & Período de coleta \\
\hline \multicolumn{7}{|l|}{ Região Metropolitana do Recife } \\
\hline Açude de Apipucos & $08^{\circ} 01^{\prime} 14^{\prime \prime} \mathrm{S} \mathrm{e}$ & Recife & eutrófico & 5 & 65 & 08/1981 a 07/1982 \\
\hline & $34^{\circ} 56^{\prime} 00^{\prime \prime} \mathrm{W}$ & & & 7 & 65 & 08/1981 a $07 / 1982$ \\
\hline Viveiros de cultivo de camarão & $\begin{array}{l}08^{\circ} 17^{\prime} 00^{\prime \prime} \mathrm{S} \mathrm{e} \\
35^{\circ} 00^{\prime} 00^{\prime \prime} \mathrm{W}\end{array}$ & Cabo & N.I. & 2,3 & 65 & 01 a $06 / 1980$ \\
\hline Reservatório de Botafogo & $\begin{array}{l}07^{\circ} 50^{\prime} 23^{\prime \prime} \mathrm{S} \mathrm{e} \\
35^{\circ} 01^{\prime} 50^{\prime \prime} \mathrm{W}\end{array}$ & Igarassu & oligotrófico & 13 & 45 & $01 / 2004$ \\
\hline Viveiros de cultivo de camarão & $\begin{array}{l}07^{\circ} 51^{\prime} 00 ”, \mathrm{~S} \mathrm{e} \\
34^{\circ} 51^{\prime} 00^{\prime \prime} \mathrm{W}\end{array}$ & Igarassu & mesotrófico & 4 & 65 & 09/1980 a 07/1981 \\
\hline Reservatório de Duas Unas & $\begin{array}{l}08^{\circ} 05^{\prime} 31^{\prime \prime} \mathrm{S} \mathrm{e} \\
35^{\circ} 02^{\prime} 19^{\prime \prime} \mathrm{W}\end{array}$ & $\begin{array}{l}\text { Jaboatão dos } \\
\text { Guararapes }\end{array}$ & eutrófico & 16 & 40 & 08/2004 \\
\hline \multicolumn{7}{|l|}{ Zona da Mata } \\
\hline Reservatório de Carpina & $\begin{array}{l}07^{\circ} 52^{\prime} 00^{\prime \prime} \mathrm{S} \mathrm{e} \\
35^{\circ} 18^{\prime} 00^{\prime \prime} \mathrm{W}\end{array}$ & Carpina & eutrófico & 10 & 125 & 07 a $12 / 2001$ \\
\hline Reservatório de Tapacurá & $\begin{array}{l}08^{\circ} 02^{\prime} 14 " \mathrm{~S} \text { e } \\
35^{\circ} 09^{\prime} 46^{\prime \prime} \mathrm{W}\end{array}$ & $\begin{array}{l}\text { São Lourenço } \\
\text { da Mata }\end{array}$ & eutrófico & 14 & 64 & 08/2003 e $01 / 2004$ \\
\hline \multicolumn{7}{|l|}{ Agreste } \\
\hline Reservatório de Jucazinho & $\begin{array}{l}07^{\circ} 58^{\prime} 02 ” \mathrm{~S} \text { e } \\
35^{\circ} 44^{\prime} 33^{\prime \prime} \mathrm{W}\end{array}$ & Surubim & oligotrófico & $\begin{array}{c}11,12 \\
15\end{array}$ & $\begin{array}{l}125 \\
125\end{array}$ & $\begin{array}{l}07 / 2001 \text { a } 08 / 2002 \\
07 / 2001 \text { a } 05 / 2002\end{array}$ \\
\hline Reservatório de Mundaú & $\begin{array}{l}08^{\circ} 57^{\prime} 17^{\prime \prime} \mathrm{S} \mathrm{e} \\
36^{\circ} 29^{\prime} 55^{\prime} \mathrm{W}\end{array}$ & Garanhuns & eutrófico & 16 & 40 & $07 / 2004$ \\
\hline Reservatório de Arcoverde & $\begin{array}{l}08^{\circ} 33^{\prime} 33^{\prime \prime} \mathrm{S} \mathrm{e} \\
36^{\circ} 59^{\prime} 07^{\prime \prime} \mathrm{W}\end{array}$ & Arcoverde & eutrófico & 16 & 40 & $07 / 2004$ \\
\hline \multicolumn{7}{|l|}{ Sertão } \\
\hline Trecho do rio São Francisco & N.I. & Orocó & oligo-mesotrófico & 8 & 50 & $11 / 1998$ \\
\hline Rio Brígida & N.I. & Orocó & oligo-mesotrófico & 8 & 50 & $11 / 1998$ \\
\hline Reservatório de Itaparica & N.I. & Floresta & eutrófico & 8 & 50 & $11 / 1998$ \\
\hline $\begin{array}{l}\text { Reservatório de Itaparica } \\
\text { (antes do represamento) }\end{array}$ & N.I. & Floresta & oligotrófico & 6 & 65 & 09 e $10 / 1987$ \\
\hline Açude de Poço da Cruz & $\begin{array}{l}08^{\circ} 30^{\prime} 31^{\prime \prime} \mathrm{S} \mathrm{e} \\
37^{\circ} 42^{\prime} 17^{\prime \prime} \mathrm{W}\end{array}$ & Ibimirim & eutrófico & $\begin{array}{c}8 \\
16\end{array}$ & $\begin{array}{l}50 \\
40\end{array}$ & $\begin{array}{l}11 / 1998 \\
07 / 2005\end{array}$ \\
\hline $\begin{array}{l}\text { Poço d'água da Faz. } \\
\text { São Gonçalo }\end{array}$ & N.I. & Custódia & N.I. & 1 & N.I. & 02/1934 a 03/1935* \\
\hline Reservatório da Ingazeira & $\begin{array}{l}08^{\circ} 34^{\prime} 00^{\prime \prime} \mathrm{S} \text { e } \\
36^{\circ} 52^{\prime} 00^{\prime \prime} \mathrm{W}\end{array}$ & Ingazeira & eutrófico & 9 & 50 & 01/1997 a 03/1998 \\
\hline Reservatório de Jazigo & $\begin{array}{l}07^{\circ} 59^{\prime} 58^{\prime \prime} \mathrm{S} \text { e } \\
38^{\circ} 14^{\prime} 31^{\prime \prime} \mathrm{W}\end{array}$ & Pajeú & eutrófico & 16 & 40 & $07 / 2005$ \\
\hline Reservatório do Saco & $\begin{array}{l}07^{\circ} 56^{\prime} 43 ”, \mathrm{~S} \text { e } \\
38^{\circ} 17^{\prime} 09^{\prime \prime} \mathrm{W}\end{array}$ & Brígida & eutrófico & 16 & 40 & $07 / 2005$ \\
\hline Reservatório de Belém & N.I. & $\begin{array}{l}\text { Belém do } \\
\text { São Francisco }\end{array}$ & oligotrófico & 6 & 65 & 09 e $10 / 1987$ \\
\hline
\end{tabular}

*Período total de coleta informado no artigo, não havendo referência da data de coleta para o local. 1) Ahlstrom (1938); 2) Neumann-Leitão (1981);

3) Paranaguá \& Neumann-Leitão (1982); 4) Neumann-Leitão \& Nogueira (1986); 5) Neumann-Leitão \& Souza (1987); 6) Neumann-Leitão \& Nogueira-Paranhos (1987/89); 7) Neumann-Leitão et al. (1989); 8) Crispim \& Watanabe (2000); 9) Bouvy et al. (2001); 10) Almeida (2002); 11) Melo Júnior (2002); 12) Melo Júnior (dados não publicados); 13) Moura (2004); 14) Almeida et al. (2006); 15) Melo Júnior (dados não publicados); e 16) Almeida (dados não publicados).

planctônicos são bastante escassos. Para Bozelli \& Huszar (2003), este fato emerge da tradição que vem de regiões temperadas de se estudar ecossistemas lênticos, principalmente lagos, onde existem grupos de pesquisa mais consolidados.

Pelo levantamento bibliográfico realizado, a fauna de rotíferos dos ecossistemas límnicos de Pernambuco está representada por 64 espé- cies, distribuídas em 18 famílias e 29 gêneros (Tabela 3). Além disso, outros 12 táxons foram referidos apenas como morfotipos: Rotaria sp., Keratella sp., Lecane sp., Cephalodella sp., Notommata sp., Trichocerca sp., Ascomorpha sp., Polyarthra sp., Synchaeta sp., Testudinella sp., Filinia sp. e Proales sp. As famílias mais representativas foram Lecanidae (21 táxons), Brachionidae (20) e Synchaetidae (4), sendo 
Tabela 2. Percentual dos assuntos abordados nos trabalhos que contemplam a fauna de rotíferos planctônicos dos ambientes aquáticos continentais de Pernambuco.

Table 2. Subjects (in percentage) covered in research about planktonic rotiferan fauna from some freshwater ecosystems in the state of Pernambuco (Brazil).

\begin{tabular}{lcc}
\hline \multicolumn{1}{c}{ Assunto abordado } & \% & Número das citações (ver referências na Tabela 1) \\
\hline Aspectos Sazonais & 81,25 & $2,3,4,5,6,7,9,10,11,12,13,14,15$ \\
Estado Trófico do Ecossistema & 75,00 & $4,5,6,7,8,9,10,11,12,14,15,16$ \\
Parâmetros Abióticos & 68,75 & $1,4,5,7,8,9,11,12,14,15,16$ \\
Densidade & 62,50 & $5,6,7,8,9,12,13,13,14,16$ \\
Caracterização Morfológica & 62,55 & $1,2,3,4,5,7,9,10,11,13$ \\
Variação Espacial & 37,50 & $5,6,7,8,14,15$ \\
Diversidade e Eqüitabilidade & 31,25 & $8,12,13,14,16$ \\
Variação Nictimeral & 25,00 & $6,13,14,15$ \\
\hline
\end{tabular}

Tabela 3. Inventário das espécies de rotíferos planctônicos dos ecossistemas aquáticos continentais de Pernambuco, com a indicação dos locais onde cada espécie foi registrada e dos códigos das referências consultadas (ver Tabela 1).

Table 3. Inventory of the planktonic rotifers species from some freshwater ecosystems in Pernambuco State (Brazil), indicating their occurrence and the respective literature source (see Table 1 ).

\begin{tabular}{|c|c|c|c|c|c|c|c|c|c|c|c|c|c|c|c|c|c|c|c|c|}
\hline & Met & $\begin{array}{r}\mathbf{R e} \\
\mathbf{r o p} \\
\mathbf{R}\end{array}$ & $\begin{array}{l}\text { giã } \\
\text { olit: } \\
\text { ecif }\end{array}$ & $\begin{array}{l}o \\
\text { ana } \\
e\end{array}$ & do & $\begin{array}{r}\mathrm{Zo} \\
\mathrm{d} \\
\mathrm{Mr}\end{array}$ & $\begin{array}{l}\text { na } \\
\text { a } \\
\text { ata }\end{array}$ & & Agre & ste & & & & & Sert & tão & & & & \\
\hline & 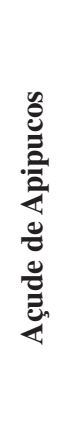 & 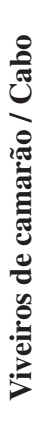 & 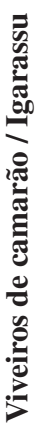 & 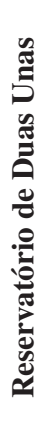 & 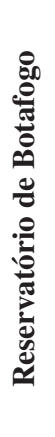 & 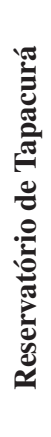 & 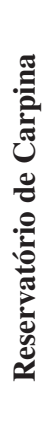 & 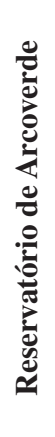 & 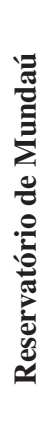 & 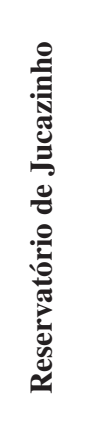 & 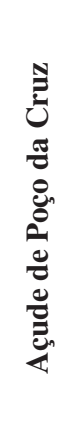 & 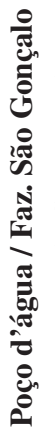 & 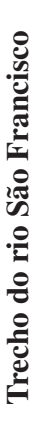 & 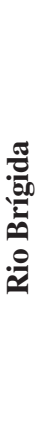 & 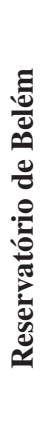 & 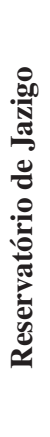 & 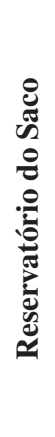 & 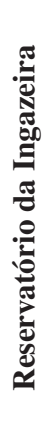 & 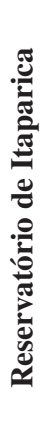 & 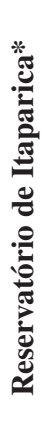 \\
\hline Código da Referência & 5,7 & 2 & 4 & 16 & 13 & 14 & 10 & 16 & 16 & 12,15 & 8,16 & 1 & 8 & 8 & 6 & 16 & 16 & 9 & 8 & 6 \\
\hline FAMÍLIA PHILODINIDAE & - & - & - & - & - & - & - & - & - & - & - & - & - & - & - & - & - & - & - & - \\
\hline Rotaria rotatoria (Pallas, 1766) & $\mathrm{X}$ & - & - & - & - & - & - & - & - & - & - & - & - & - & $\mathrm{x}$ & - & - & - & - & $\mathrm{X}$ \\
\hline Rotaria sp. & $\mathrm{X}$ & - & - & - & - & $\mathrm{X}$ & - & - & - & - & - & - & $\mathrm{X}$ & - & $\mathrm{X}$ & - & - & - & - & $\mathrm{X}$ \\
\hline FAMÍLIA ASPLANCHINIDAE & - & - & - & - & - & - & - & - & - & - & - & - & - & - & - & - & - & - & - & - \\
\hline Asplanchna priodonta (Gosse, 1850) & $\mathrm{X}$ & - & - & - & - & - & - & - & - & - & - & - & - & - & - & - & - & - & - & - \\
\hline Asplanchna herrick (De Guerne, 1888) & - & - & - & - & - & - & - & - & - & - & - & - & - & - & - & - & - & - & - & $\mathrm{X}$ \\
\hline Asplanchnopus hyalinus (Harring, 1913) & - & - & - & - & - & $\mathrm{X}$ & - & - & - & - & - & - & - & - & - & - & - & - & - & - \\
\hline FAMÍLIA BRACHIONIDAE & - & - & - & - & - & - & - & - & - & - & - & - & - & - & - & - & - & - & - & - \\
\hline Platyias quadricornis (Ehrenberg, 1832) & $\mathrm{X}$ & $\mathrm{X}$ & - & - & - & $\mathrm{x}$ & - & - & - & - & - & - & $\mathrm{x}$ & $\mathrm{X}$ & $\mathrm{X}$ & - & - & - & - & $\mathrm{X}$ \\
\hline Plationus patulus (O. F. Müller, 1786) & $\mathrm{X}$ & $\mathrm{x}$ & - & - & - & - & $\mathrm{X}$ & - & - & $\mathrm{X}$ & $\mathrm{X}$ & - & - & - & - & - & - & - & $\mathrm{x}$ & $\mathrm{X}$ \\
\hline Brachionus quadridentatus (Hermann, 1783) & $\mathrm{X}$ & - & - & - & - & $\mathrm{x}$ & - & - & - & - & - & - & $\mathrm{x}$ & - & - & - & - & - & - & - \\
\hline Brachionus calyciflorus (Pallas, 1766) & $\mathrm{X}$ & - & - & - & - & $\mathrm{X}$ & $\mathrm{x}$ & - & $\mathrm{X}$ & $\mathrm{X}$ & - & - & - & - & - & $\mathrm{X}$ & - & $\mathrm{X}$ & - & - \\
\hline Brachionus dolabratus (Harring, 1915) & - & - & - & - & - & $\mathrm{X}$ & - & - & - & - & - & - & - & - & - & - & - & - & - & - \\
\hline Brachionus falcatus (Zacharias, 1898) & $\mathrm{X}$ & $\mathrm{X}$ & - & - & $\mathrm{X}$ & $\mathrm{X}$ & $\mathrm{X}$ & - & $\mathrm{x}$ & $\mathrm{X}$ & - & - & - & - & - & $\mathrm{X}$ & $\mathrm{x}$ & $\mathrm{X}$ & - & - \\
\hline Brachionus havanaensis (Rousselet, 1911) & - & - & - & - & - & $\mathrm{X}$ & $\mathrm{X}$ & $\mathrm{X}$ & - & $\mathrm{X}$ & - & - & $\mathrm{x}$ & - & - & - & - & - & - & - \\
\hline Brachionus angularis (Gosse, 1851) & $\mathrm{X}$ & - & - & $\mathrm{X}$ & - & - & - & - & - & $\mathrm{X}$ & - & - & - & - & - & - & - & $\mathrm{X}$ & - & - \\
\hline Brachionus caudatus (Barrois \& Daday, 1894) & $\mathrm{X}$ & $\mathrm{X}$ & - & - & - & - & - & - & - & - & - & - & - & - & - & - & - & - & - & - \\
\hline Brachionus rubens (Ehrenberg, 1838) & - & - & - & - & $\mathrm{X}$ & - & $\mathrm{x}$ & - & - & $\mathrm{X}$ & - & $\mathrm{X}$ & - & - & - & - & - & $\mathrm{X}$ & - & - \\
\hline Brachionus urceolaris (O. F. Muller, 1773) & - & - & - & - & - & - & - & - & - & - & $\mathrm{X}$ & - & - & - & - & - & - & - & - & - \\
\hline Keratella tropica (Apstein, 1907) & $\mathrm{X}$ & - & - & $\mathrm{x}$ & - & $\mathrm{X}$ & $\mathrm{x}$ & - & $\mathrm{x}$ & $\mathrm{x}$ & $\mathrm{X}$ & - & $\mathrm{X}$ & $\mathrm{X}$ & - & $\mathrm{x}$ & - & $\mathrm{x}$ & - & - \\
\hline Keratella americana Carlin, 1943 & - & - & - & $\mathrm{x}$ & $\mathrm{x}$ & $\mathrm{x}$ & - & - & - & - & - & - & - & - & $\mathrm{x}$ & $\mathrm{x}$ & - & - & - & $\mathrm{x}$ \\
\hline
\end{tabular}


Tabela 3. Continuação...

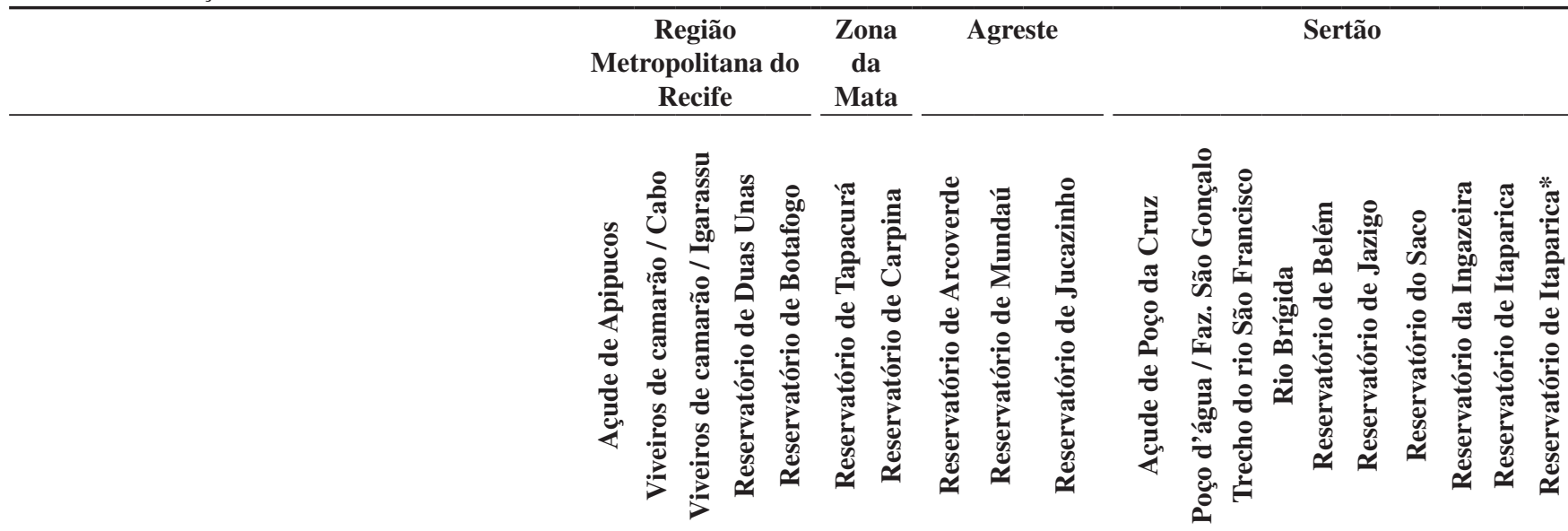

\begin{tabular}{|c|c|c|c|c|c|c|c|c|c|c|c|c|c|c|c|c|c|c|c|c|}
\hline Código da Referência & 5,7 & 2 & 4 & 16 & 13 & 14 & 10 & 16 & 16 & 12,15 & 8,16 & 1 & 8 & 8 & 6 & 16 & 16 & 9 & 8 & 6 \\
\hline Keratella cochlearis (Gosse, 1851) & - & - & - & - & $\mathrm{x}$ & - & - & - & - & - & - & - & - & - & $\mathrm{x}$ & - & - & - & - & $\mathrm{x}$ \\
\hline Keratella thomassoni (Hauer, 1958) & - & - & - & - & - & - & - & - & - & - & - & - & $\mathrm{x}$ & - & - & - & - & - & - & - \\
\hline Keratella sp. & - & - & - & - & - & - & - & - & - & - & - & - & - & - & - & - & - & $\mathrm{x}$ & - & - \\
\hline Trichotria tetractis (Ehrenberg, 1830) & - & - & - & - & - & - & - & - & - & - & - & - & - & - & $\mathrm{x}$ & - & - & - & - & $\mathrm{x}$ \\
\hline Macrochaetus ongipes (Myers, 1934) & - & - & - & - & - & - & - & - & - & - & - & - & - & $\mathrm{x}$ & - & - & - & - & - & - \\
\hline Macrochaetus collinsi (Gosse, 1867) & - & - & - & - & - & - & - & - & - & - & - & - & - & - & $\mathrm{x}$ & - & - & - & - & $\mathrm{x}$ \\
\hline Anuraeopsis fissa (Gosse, 1851) & - & - & - & - & - & $\mathrm{x}$ & - & - & - & - & - & - & - & - & $\mathrm{x}$ & - & - & - & - & $\mathrm{x}$ \\
\hline
\end{tabular}

FAMÍLIA EPIPHANIDAE

Epiphanes macrouros

(Barrois \& Daday, 1894)

Epiphanes clavulata (Ehrenberg, 1832)

FAMÍLIA EUCHLANIDAE

Euchlanis incisa (Carlin, 1939)

Euchlanis dilatata (Ehrenberg, 1832)

FAMÍLIA GASTROPODIDAE

Ascomorpha sp.

FAMÍLIA LECANIDAE

Lecane imbricata (Carlin, 1939)

Lecane clara (Bryce, 1892)

Lecane ludwigi (Eckstein, 1883)

Lecane curvicornis (Murray, 1913)

Lecane papuana (Murray, 1913)

Lecane luna (O.F.Muller, 1776)

Lecane lunaris (Ehrenberg, 1832)

Lecane bulla (Gosse, 1886)

Lecane mira (Murray, 1913)

Lecane ohioensis (Herrick, 1885)

Lecane tudicola (Harry\& Myers, 1926)

Lecane unguitata (Wiszniewski, 1954)

Lecane leontina (Turner, 1892)

Lecane scutata (Harring \& Myers, 1926)

Lecane aculeata (Jakubski, 1912)

Lecane cornuta (O. F. Müller, 1786)

Lecane obtusa (Murray, 1913)

Lecane hornemanni (Ehrenberg, 1834)

Lecane stichaea (Harring, 1913)

Lecane ornata (Voigt, 1957) 
Tabela 3. Continuação.

\begin{tabular}{|c|c|c|c|c|c|c|c|c|c|c|c|c|c|c|c|c|c|c|c|c|}
\hline & \multicolumn{5}{|c|}{$\begin{array}{c}\text { Região } \\
\text { Metropolitana do } \\
\text { Recife } \\
\end{array}$} & \multicolumn{2}{|c|}{$\begin{array}{c}\text { Zona } \\
\text { da } \\
\text { Mata } \\
\end{array}$} & \multicolumn{3}{|c|}{ Agreste } & \multicolumn{10}{|c|}{ Sertão } \\
\hline & 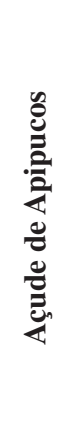 & 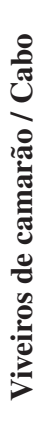 & 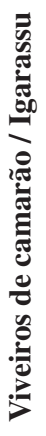 & 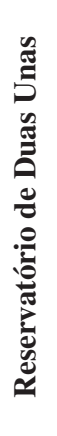 & 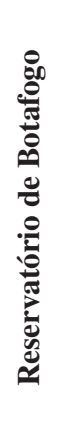 & 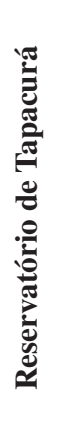 & 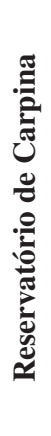 & 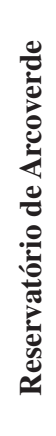 & 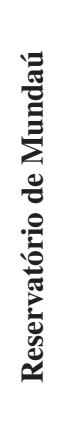 & 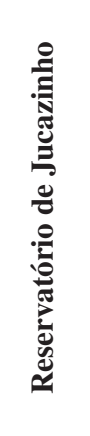 & 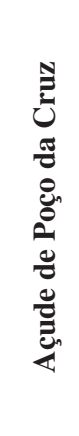 & 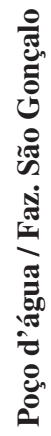 & 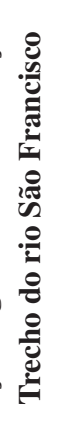 & $\begin{array}{l}0 \\
0 \\
0\end{array}$ & 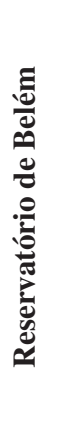 & 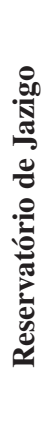 & 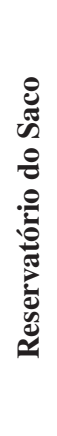 & 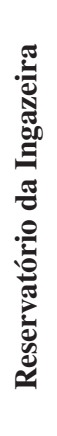 & 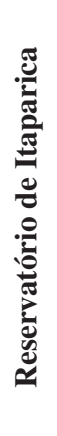 & 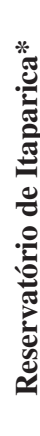 \\
\hline Código da Referência & 5,7 & 2 & 4 & 16 & 13 & 14 & 10 & 16 & 16 & 12,15 & 8,16 & 1 & 8 & 8 & 6 & 16 & 16 & 9 & 8 & 6 \\
\hline Lecane sp. & - & - & - & - & - & $\mathrm{x}$ & - & - & - & - & - & - & - & - & - & - & - & - & - & - \\
\hline FAMÍLIA LEPADELLIDAE & - & - & - & - & - & - & - & - & - & - & - & - & - & - & - & - & - & - & - & - \\
\hline Lepadella patella (O. F. Müller, 1786) & - & - & - & - & - & $\mathrm{x}$ & - & - & - & - & - & - & - & - & - & - & - & - & - & $\mathrm{x}$ \\
\hline Lepadella akrobeles (Myers, 1934) & - & - & - & - & - & - & - & - & - & - & - & - & - & $\mathrm{x}$ & - & - & - & - & - & - \\
\hline Lepadella cf. ovalis (O. F. Müller, 1786) & - & - & $\mathrm{x}$ & - & - & - & - & - & - & - & - & - & $\mathrm{x}$ & - & - & - & - & - & - & - \\
\hline FAMÍLIA MYTILLINIDAE & - & - & - & - & - & - & - & - & - & - & - & - & - & - & - & - & - & - & - & - \\
\hline Mytilina ventralis (Ehrenberg, 1832) & - & - & - & - & - & - & - & - & - & - & - & - & $\mathrm{x}$ & - & - & - & - & - & - & - \\
\hline Lophocharis salpina (Ehrenberg, 1834) & - & - & - & - & - & - & - & - & - & - & - & - & - & - & $\mathrm{x}$ & - & - & - & - & - \\
\hline FAMÍLIA NOTOMMATIDAE & - & - & - & - & - & - & - & - & - & - & - & - & - & - & - & - & - & - & - & - \\
\hline Cephalodella sp. & - & $\mathrm{x}$ & - & - & - & $\mathrm{x}$ & - & - & - & - & - & - & - & - & $\mathrm{x}$ & - & - & - & - & - \\
\hline Notommata sp. & - & - & - & - & - & - & - & - & - & - & - & - & - & - & - & - & - & - & - & $\mathrm{x}$ \\
\hline FAMÍLIA PROALIDAE & - & - & - & - & - & - & - & - & - & - & - & - & - & - & - & - & - & - & - & - \\
\hline Proales sp. & - & - & - & - & - & - & - & - & - & - & - & - & - & - & - & - & - & - & - & $\mathrm{x}$ \\
\hline FAMÍLIA SCARIDIIDAE & - & - & - & - & - & - & - & - & - & - & - & - & - & - & - & - & - & - & - & - \\
\hline Scaridium longicaudum (Müller, 1786) & - & - & - & - & - & - & - & - & - & - & - & - & - & $\mathrm{x}$ & - & - & - & - & - & - \\
\hline FAMÍLIA SYNCHAETIDAE & - & - & - & - & - & - & - & - & - & - & - & - & - & - & - & - & - & - & - & - \\
\hline Ploesoma truncatum (Levander, 1894) & - & - & - & - & - & $\mathrm{x}$ & - & - & $\mathrm{x}$ & - & - & - & - & - & $\mathrm{x}$ & - & - & - & - & $\mathrm{x}$ \\
\hline Polyarthra vulgaris (Carlin, 1943) & $\mathrm{x}$ & - & - & - & - & - & - & $\mathrm{x}$ & $\mathrm{x}$ & - & - & - & - & - & - & $\mathrm{x}$ & - & - & - & - \\
\hline Polyarthra sp. & - & - & - & - & - & - & - & - & - & - & - & - & - & - & - & - & - & $\mathrm{x}$ & - & - \\
\hline Synchaeta sp. & - & - & - & - & - & - & - & - & - & - & - & - & - & - & - & - & - & - & - & $\mathrm{x}$ \\
\hline FAMÍLIA TRICHOCERCIDAE & - & - & - & - & - & - & - & - & - & - & - & - & - & - & - & - & - & - & - & - \\
\hline Trichocerca pusilla (Lauterborn, 1898) & - & - & - & - & - & - & - & - & - & - & - & - & - & - & - & - & - & - & - & $\mathrm{x}$ \\
\hline Trichocerca cylindrica (Imhof, 1891) & - & - & - & - & - & - & - & - & - & - & - & - & - & - & - & - & - & - & - & $\mathrm{x}$ \\
\hline Trichocerca sp. & - & - & $\mathrm{x}$ & - & - & $\mathrm{x}$ & - & - & - & - & - & - & - & - & - & - & - & $\mathrm{x}$ & - & - \\
\hline FAMÍLIA TROCHOSPHAERIDAE & - & - & - & - & - & - & - & - & - & - & - & - & - & - & - & - & - & - & - & - \\
\hline Filinia longiseta (Ehrenberg, 1834) & $\mathrm{x}$ & $\mathrm{x}$ & - & $\mathrm{x}$ & $\mathrm{x}$ & - & - & - & - & $\mathrm{x}$ & - & - & - & $\mathrm{x}$ & - & - & - & - & - & - \\
\hline Filinia opoliensis (Zacharias, 1891) & - & - & - & - & $\mathrm{x}$ & - & - & $\mathrm{x}$ & $\mathrm{x}$ & - & - & - & - & - & - & - & - & - & - & - \\
\hline Filinia sp. & - & $\mathrm{x}$ & - & - & - & - & - & - & - & - & - & - & - & - & - & - & - & $\mathrm{x}$ & - & - \\
\hline FAMÍLIA FLOSCULARIIDAE & - & - & - & - & - & - & - & - & - & - & - & - & - & - & - & - & - & - & - & - \\
\hline Lacinularia striolata (Shephard, 1899) & - & - & - & - & - & - & - & - & - & - & - & $\mathrm{x}$ & - & - & - & - & - & - & - & - \\
\hline FAMÍLIA HEXARTHRIDAE & - & - & - & - & - & - & - & - & - & - & - & - & - & - & - & - & - & - & - & - \\
\hline Conochilus unicornis (Rousselet, 1892) & - & - & - & - & - & $\mathrm{x}$ & - & - & - & - & - & - & - & - & - & - & - & - & - & - \\
\hline Conochilus dossuarius (Hudson, 1885) & $\mathrm{x}$ & - & - & - & - & - & - & - & - & - & - & - & - & - & $\mathrm{x}$ & - & - & - & - & $\mathrm{x}$ \\
\hline Hexarthra fennica (Levander, 1892) & - & - & - & $\mathrm{x}$ & - & - & - & - & $\mathrm{x}$ & - & - & - & - & - & - & $\mathrm{x}$ & $\mathrm{x}$ & - & - & - \\
\hline FAMÍLIA TESTUDINELLIDAE & - & - & - & - & - & - & - & - & - & - & - & - & - & - & - & - & - & - & - & - \\
\hline Testudinella patina (Hermann, 1783) & $\mathrm{x}$ & - & - & - & - & - & - & - & - & - & - & - & - & - & - & $\mathrm{x}$ & - & - & - & - \\
\hline Testudinella sp. & - & - & - & $\mathrm{x}$ & - & - & - & - & - & - & - & - & - & - & - & - & - & - & - & - \\
\hline
\end{tabular}


as duas primeiras as mais diversificadas nos trópicos (Segers 1995). Dentre os gêneros registrados em Pernambuco, destacam-se Lecane (21 táxons), Brachionus (9) e Keratella (5). No Brasil são conhecidas 112 espécies de Lecanidae e 42 espécies de Brachionidae. Espera-se, entretanto, que o número de espécies dessas e de outras famílias possa aumentar bastante, quando forem realizados inventários mais completos com maior cobertura geográfica das regiões Amazônica, Nordeste e Centro-oeste (Rocha, dados não publicados).

A maioria dos trabalhos utilizou rede com 65 micrômetros de abertura de malha (Tabela 1), entretanto, estudos realizados no Brasil têm demonstrado que um maior número de espécies de rotíferos planctônicos límnicos é registrado quando redes com abertura de malha menor que esta são utilizadas (Lucinda et al. 2004, Almeida et al. 2006, Rocha, dados não publicados). A riqueza de rotíferos de Pernambuco é aparentemente bastante inferior à encontrada em outros Estados brasileiros, tais como Mato Grosso (Neves et al. 2003), Paraná (Ulloa 2004) e Minas Gerais (Eskinazi-Sant' Anna et al. 2005). Em Mato Grosso, Neves et al. (2003) registraram 70 espécies (além de 9 táxons referidos sem o epíteto específico) para apenas duas lagoas marginais do rio Cuiabá. Eskinazi-Sant' Anna et al. (2005), em um trabalho preliminar de revisão bibliográfica considerando todo estado de Minas Gerais, registraram 300 espécies de rotíferos planctônicos para 59 ambientes aquáticos. Para o Paraná, Ulloa (2004) encontrou 88 espécies de rotíferos planctônicos em diversos ecossistemas da Bacia do Alto Rio Paraná, cuja biodiversidade total de rotíferos é estimada em cerca de 230 espécies (Aoyagui \& Bonecker 2004). Entretanto, Rocha (dados não publicados) aponta a relação entre o maior número de ocorrências registradas nas áreas onde se concentra o maior número de pesquisadores dedicados a estudos taxonômicos de grupos planctônicos continentais, o que poderia ser uma das causas para esta baixa riqueza de espécies de rotíferos em Pernambuco. Além disso, algumas ameaças potenciais também poderiam estar afetando drasticamente a biodiversidade de rotíferos não só do estado de Pernambuco, mas também em boa parte da região Nordeste, incluindo o elevado represamento de sistemas lóticos ainda pouco estudados, os múltiplos usos dos sistemas lênticos naturais e artificiais, a eutrofização crescente destes locais e, mais recentemente, as obras de transposição do rio São Francisco.

Considerando a freqüência de ocorrência das espécies nos 19 locais estudados, apenas Keratella tropica e Brachionus falcatus, ambas da família Brachionidae, apresentaram valores percentuais maiores ou iguais a $50 \%$ (1). A alta freqüência destas espécies confirma a grande importância destes rotíferos no zooplâncton de águas continentais tropicais. Keratella tropica é uma espécie planctônica tropical (Dábes 1995, Starling 2000, Duggan et al. 2001, Leitão et al. e Vitório et al., ambos dados não publicados). Almeida (2005), através de revisão de literatura, também cita a presença desta espécie em ecossistemas lênticos dos estados do Ceará, Paraíba, Minas Gerais e São Paulo. A espécie Brachionus falcatus está distribuída nas regiões tropicais e subtropicais, ocorrendo em águas doces e salobras (Neumann-Leitão \& Souza 1987, Reid \& Turner 1988). Também foi registrada para açudes, reservatórios e lagoas dos estados do Maranhão, Paraíba, Goiás, Mato Grosso do Sul, Minas Gerais, São Paulo e Rio Grande do Sul (ver revisão de Almeida 2005). Ambas as espécies habitam ambientes de oligotróficos a eutróficos.

Doze outras espécies de rotíferos foram consideradas acessórias, representando 16,88\% do total. Entre essas espécies, a maior parte pertence às famílias Brachionidae e Lecanidae, além da ocorrência das famílias Philodinidae, Epiphanidae e Trochosphaeridae. A grande maioria dos rotíferos $(80,52 \%)$ foi considerada acidental quanto à ocorrência. Esse alto percentual de espécies acidentais revelou que, até o momento, a maioria das populações de rotíferos do estado de Pernambuco é exclusiva para determinados locais. Além disso, foi

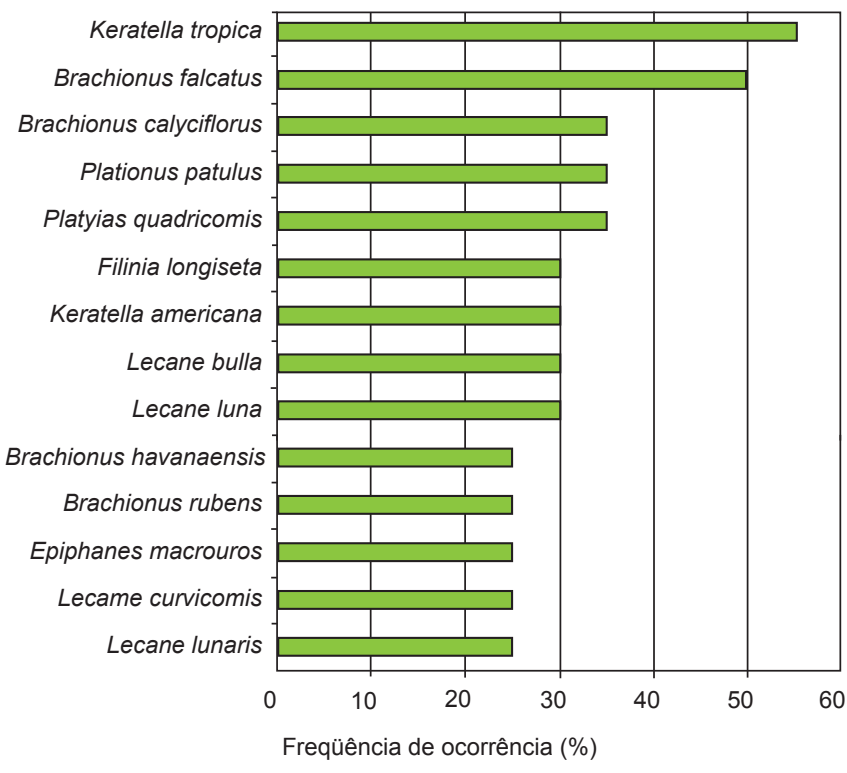

Figura 1. Frequiência de ocorrência das espécies de rotíferos planctônicos de ecossistemas aquáticos continentais de Pernambuco, com percentuais maiores ou iguais a $25 \%$.

Figure 1. Occurrence of the most representative planktonic rotifers species (over $25 \%$ of the overall occurrence) from some freshwater environments in Pernambuco State (Brazil).

observado um maior direcionamento das pesquisas para a zona limnética dos ecossistemas estudados, visto que as espécies da família Brachionidae apresentaram maiores freqüências e são características das regiões abertas (limnéticas) dos ecossistemas aquáticos continentais (Hutchinson 1967, Neumann-Leitão \& Nogueira 1986).

A baixa frequiência observada para as espécies da família Lecanidae pode ser reflexo de vários fatores e, dentre as prováveis causas, podem ser destacadas: (1) a difícil resolução quanto à identificação taxonômica desse grupo de rotíferos, visto que as espécies desta família têm sido alvo de muita discussão entre os especialistas, o que pode ser traduzida em identificações errôneas ou incompletas; e (2) a pouca ênfase dada à distribuição horizontal dos corpos d' água estudados, com poucas coletas realizadas na zona litorânea dos locais. As espécies desta família possuem normalmente hábito bentônico, embora possam ser registradas freqüentemente no plâncton, estando principalmente relacionadas às regiões litorâneas dos locais límnicos, sobretudo em locais ricos em vegetação (Hutchinson 1967, Neumann-Leitão \& Nogueira 1986). Boa parte dos estudos realizados em Pernambuco menciona a presença de bancos de macrófitas nos locais estudados. Entretanto, apenas um dos trabalhos catalogados apresentou coletas na região litorânea com influência de macrófitas (Almeida et al 2006), resultando em um dos maiores levantamentos de espécies desta família no Estado.

O conhecimento dos rotíferos no estado de Pernambuco apresenta várias limitações, principalmente quanto às dificuldades taxonômicas peculiares a este grupo e ao acesso aos diversos ecossistemas, alguns dos quais temporários. A escassez de estudos que alberguem ciclos hidrológicos inteiros (anuais) e as limitações espaciais desses estudos (tanto horizontais quanto verticais) mostram-se como grandes desafios aos pesquisadores no que diz respeito ao conhecimento real da biodiversidade de rotíferos do Estado, sendo estas importantes lacunas a serem preenchidas em estudos futuros.

Neste contexto, o presente trabalho se mostra importante na avaliação e síntese da biodiversidade de rotíferos do Estado, servindo como base para estudos futuros de monitoramento de bacias ou implementa- 
ção de programas de conservação da biodiversidade de espécies ou de ecossistemas. Sugere-se, desta forma, que um maior direcionamento das pesquisas sobre este grupo de organismos seja realizado para ambientes localizados na Zona da Mata e no Agreste, e em ecossistemas lóticos. Outras áreas a serem contempladas em estudos futuros sobre a biodiversidade de rotíferos no Estado consistem na caracterização das zonas litorâneas dos ecossistemas e no inventário de espécies típicas de ambientes límnicos temporários. Os resultados obtidos a partir deste inventário bibliográfico contribuem para o conhecimento da biodiversidade e distribuição de espécies no Brasil em diferentes biomas.

\section{Agradecimentos}

Este trabalho foi parcialmente financiado pelo $\mathrm{CNPq}$, através de bolsas de Iniciação Científica ao primeiro autor (PIBIC/UFRPE/CNPq - 2001/2002) e de mestrado a segunda autora (CNPq/UFPE/PPG em Biologia Animal-2003/2005). Agradecemos à Dra. Tâmara de Almeida e Silva (UNEB), ao MSc. Arthur Ziggiatti Güth (IO/USP) e aos biólogos Alexandre de Melo Medeiros (SECEDUCA, Recife) e Márcio Hidekazu Ohkawara (IO/USP) pelas valiosas revisões, críticas e sugestões ao manuscrito. Gostaríamos ainda de agradecer aos três revisores anônimos pelas sugestões e críticas construtivas.

\section{Referências Bibliográficas}

AHLSTROM, E.H. 1938. Plankton Rotatoria from Northeast Brazil. Ann. Acad. Bras. Cienc. 10(1):29-51.

ALMEIDA, V.L.S. 2002. Biodiversidade da comunidade zooplanctônica do reservatório de Carpina, Zona da Mata, Pernambuco - Brasil. Monografia de Graduação, Universidade Federal Rural de Pernambuco, Recife.

ALMEIDA, V.L.S. 2005. Ecologia do zooplâncton do reservatório de Tapacurá, Pernambuco - Brasil. Dissertação de Mestrado, Universidade Federal de Pernambuco, Recife.

ALMEIDA, V.L.S., LARRAZÁBAL, M.E.L., MOURA, A.N., MELO JÚNIOR, M. 2006. Rotifera das zonas limnética e litorânea do reservatório de Tapacurá, Pernambuco, Brasil. Iheringia, Sér. Zool. 96(4):445-451.

AOYAGUI, A.S.M. \& BONECKER, C.C. 2004. The art status of rotifers studies in natural environments of South América: floodplains. Acta Sci. Biol. Sci. 26(4): 385-406.

BARNES, R.S.K., CALOW, P. \& OLIVE, P.J.W. 1995. Os invertebrados: uma nova síntese. Atheneu, São Paulo.

BERZINS, B. \& PEJLER, B. 1989. Rotifer ocurrence in relation to oxygen content. Hydrobiologia. 183:165-172.

BONECKER, C.C. \& AOYAGUI, A.S.M. 2005. Relationships between rotifers, phytoplankton and bacterioplankton in the Corumbá reservoir, Goiás State, Brazil. Hydrobiologia. 546:415-421.

BOUVY, M., PAGANO, M. \& TROUSSELLIER, M. 2001. Effects of a cyanobacterial bloom (Cylindrospermopsis raciborskii) on bacteria and zooplankton communities in Ingazeira Reservoir (Northeast Brazil). Aquat. Microb. Ecol. 25:215-227.

BOZELLI, R.L. \& HUSZAR, V.L.M. 2003. Comunidades fito e zooplanctônicas em tempo de avaliação. Limnotemas. 3:1-32.

CRISPIM, M.C. \& WATANABE, T. 2000. Caracterização limnológica das bacias doadoras e receptoras de águas do rio São Francisco: 1 - Zooplâncton. Acta Limnol. Bras. 12:93-103.

DÁBES, M.B.G.S. 1995. Composição e descrição do zooplâncton de 5 (cinco) lagoas marginais do rio São Francisco, Pirapora, Três Marias, Minas Gerais, Brasil. Rev. Brasil. Biol. (Braz. J. Biol.) 55(4):831-845.

DEVETTER, M. \& SED' A, J. 2003. Rotifer fecundity in relation to components of microbial food web in a eutrophic reservoir. Hydrobiologia. 504:167-175.

DUGGAN, I.C., GREEN, J.D., SHIEL, R.J. 2001. Distribution of rotifers in North island, New Zealand, and their potential use as indicator of lake trophic state. Hydrobiologia 446:155-164.
ESKINAZI-SANT' ANNA, E.M., MAIA-BARBOSA, P.M., BRITO, S. \& RIETZLER, A.C. 2005. Zooplankton biodiversity of Minas Gerais state: a preliminary synthesis of present knowledge. Acta Limnol. Bras. 17(2):199-218.

GROOMBRIDGE, B. 1992. Global biodiversity: status of the earth's living resources. Chapman \& Hall, Londres.

GÜNTZEL, A. \& ROCHA, O. 1998. Relações entre a comunidade zooplanctônica e as condições tróficas da lagoa Caconde, Osório, RS, Brasil. Iheringia, Sér. Zool. 84:65-71.

HEYWOOD, V. H. (ed). 1995. The global biodiversity assessment. Cambridge University Press, Cambridge.

HUTCHINSON, G.E. 1967. A Treatise on Limnology. II. Introduction to lake biology and their limnoplankton. John Wiley \& Sons, New York.

LUCINDA, I., MORENO, I.H, MELÃO, M.G.G. \& MATSUMURA-TUNDISI, T. 2004. Rotifers in freshwater habitats in the upper Tietê river basin, São Paulo State, Brazil. Acta. Limnol. Bras. 16(3):203-224.

MARGALEF, R. 1983. Limnologia. Omega, Barcelona.

MELO JÚNIOR, M. 2002. Ecologia e biodiversidade da fauna planctônica do reservatório de Jucazinho, Agreste de Pernambuco, Brasil. Monografia de Graduação, Universidade Federal Rural de Pernambuco, Recife.

MOURA, S.I. 2004. Zooplâncton do reservatório de Botafogo, Igarassu - PE, Brasil. Monografia de Especialização, Universidade Federal Rural de Pernambuco, Recife.

NEUMANN-LEITÃO, S. 1981. Rotíferos de Pernambuco. I Algumas espécies que ocorrem em viveiros de cultivo de camarões do Cabo - PE. An. Soc. Nordest. Zool. 3:191-199.

NEUMANN-LEITÃO, S. \& NOGUEIRA, J.D.C. 1986. Rotíferos, cladóceros e copépodos de Pernambuco. I. Algumas espécies que ocorrem em viveiros de cultivo de camarões de Nova Cruz. An. Soc. Nordest. Zool. 2(2):87-118.

NEUMANN-LEITÃO, S. \& NOGUEIRA-PARANHOS, J.D. 1987/89. Zooplâncton do Rio São Francisco - região nordeste do Brasil. Trab. Oceanogr. Univ. Fed. PE. (Tropical Oceanography) 20:173-196.

NEUMANN-LEITÃO, S., NOGUEIRA-PARANHOS, J.D. \& SOUZA, F.B.V.A. 1989. Zooplâncton do Açude de Apipucos, Recife - PE (Brasil). Arq. Biol. Tecnol. (Braz. arch. biol. technol.) 32(4):803-821.

NEUMANN-LEITÃO, S. \& SOUZA, F.B.V.A. 1987. Rotíferos planctônicos do açude de Apipucos. Recife-PE (Brasil). Arq. Biol. Tecnol. (Braz. J. Biol.) 30(3):393-418.

NEVES, I.F., ROCHA, O., ROCHE, K.F. \& PINTO, A.A. 2003. Zooplankton community structure of two marginal lakes of the River Cuiabá (Mato Grosso, Brazil) with analysis of Rotifera and Cladocera diversity. Braz. J. Biol. 63(2):329-343.

NOGUEIRA, M.G. 2001. Zooplankton composition, dominance and abundance as indicators of environmental compartimentalization in Jurumirim reservoir (Paranapanema river), São Paulo, Brazil. Hydrobiologia. 455:1-18.

NORDY, N. \& WATANABE, T. 1978. Nota preliminar sobre os rotíferos (zooplâncton) do açude Epitácio Pessoa, Boqueirão, Paraíba. Rev. Nordest. Biol. 1(1):31-39.

PARANAGUÁ, M.N. \& NEUMANN-LEITÃO, S. 1982. Rotíferos de Pernambuco. II. Espécies planctônicas que ocorrem nos viveiros de camarões do Cabo - PE. Trab. Oceanogr. Univ. Fed. PE. (Tropical Oceanography) 17:123-134.

REID, J.W. \& TURNER, P.N. 1988. Panktonic Rotifera, Copepoda and Cladocera from Açú and Viana lakes, State of Maranhão, Brazil. Rev. Bras. Biol. (Braz. J. Biol.) 48(3):485-495.

SCHÄEFER, A. 1985. Fundamentos de ecologia e biogeografia de águas continentais. Editora da UFRGS, Porto Alegre.

SEGERS, H. 1995. Rotifera: the Lecanidae (Monogononta). In Guides to the identification of the microinvertebrates of the world $(\mathrm{H}$. Dumont \& T. Nogrady, eds.), vol. 6. SPC Academic Publishing, The Hague. 
SEGERS, H. 2002. The nomenclature of the Rotifera: annoted checklist of valid family- and genus-group names. J. Nat. Hist. 36:631-640.

SIPAÚBA-TAVARES, L.H. \& ROCHA, O. 2001. Produção de plâncton (fitoplâncton e zooplâncton) para alimentação de organismos aquáticos. Rima, São Carlos.
STARLING, F.L.R.M. 2000. Comparative study of the zooplankton composition of six lacustrine ecosystems in Central Brazil during the dry season. Rev. Brasil. Biol. (Braz. J. Biol.) 60(1):101-111.

ULLOA, V. 2004. Density and biomass of planktonic rotifers in different habitats in upper Paraná River (PR, Brazil). Acta Limnol. Bras. 16(3):281-292. 
